\title{
NEW GROUNDED-CAPACITOR SINUSOIDAL OSCILLATORS USING THE CURRENT-FEEDBACK-AMPLIFIER POLE
}

\author{
MUHAMMAD TAHER ABUELMA'ATTI* \\ and HUSAIN ABDULLAH AL-ZAHER \\ King Fahd University of Petroleum and Minerals, Box 203, \\ Dhahran 31261, Saudi Arabia
}

(Received 2 November 1997; In final form 23 January 1998)

\begin{abstract}
New current-feedback-operational amplifier (CFOA)-pole-based sinusoidal oscillator circuits are presented. Each circuit uses two CFOAs, two (or three) grounded capacitors and/or resistors. Experimental results are included.
\end{abstract}

Keywords: Oscillators; current-feedback amplifier

\section{INTRODUCTION}

It is well known that oscillator circuit realization using the internal pole of the voltage feedback operational amplifier (VFOA) results in reliable high frequency performance and low component count [1-3]. The current feedback operational amplifier (CFOA) has a larger bandwidth and higher slew rate compared to the conventional VFOA. Thus the realization of oscillator circuits using the internal pole of the CFOA would result in higher frequencies of operation than their VFOA counterpart. This conjecture was examined and recently a number of oscillator circuits, exploiting to advantage the internal pole of the CFOA, have been presented [4-12]. In [4] three sinusoidal oscillator circuits using the CFOA pole are presented. While the first oscillator circuit uses two CFOAs and six resistors, the second

*Corresponding author. 
oscillator circuit uses two CFOAs and eight resistors. While both circuits use no external capacitors, they do not enjoy the attractive feature of independent control of the frequency and the condition of oscillation. Thus, while the frequency of oscillation can be controlled without disturbing the condition of oscillation, the condition of oscillation can not be controlled without disturbing the frequency of oscillation. The third oscillator circuit uses one CFOA, three resistors and one floating capacitor. In this oscillator circuit, using only four passive elements, the frequency of oscillation can not be controlled without disturbing the condition of oscillation. In [5] a multiphase active- $R$ sinusoidal oscillator circuit using the CFOA-pole is presented. Each phase requires one CFOA and two resistors; one of them is floating. Moreover, the frequency of oscillation can not be controlled without disturbing the condition of oscillation. In [6] three single-CFOA-pole-based oscillator circuits are presented. For these oscillators, the frequency of oscillation and the condition of oscillation can be independently controlled. However, each circuit requires three resistors; one of them floating, and one floating capacitor. Moreover, the control of the frequency of oscillation is achieved by using the floating resistor. The active- $R$ oscillator circuits proposed in $[7,8]$ use two CFOAs and five resistors; three of them are floating. For these oscillators the frequency of oscillation can not be controlled without disturbing the condition of oscillation. The single CFOA-pole-based oscillator circuit proposed in [9] enjoys the independent control of the frequency of oscillation and the condition of oscillation. However, it requires two grounded resistors, one floating resistor and one floating capacitor. The CFOA-pole based oscillator circuit proposed in [10] enjoys the independent control of the frequency of oscillation and the condition of oscillation. However, it uses a floating capacitor and three resistors; one of them is floating. The three sinusoidal oscillator circuits proposed in [11] exploit to advantage the internal pole of a single CFOA. Each circuit uses five externally connected passive elements; two (or three) resistors and three (or two) capacitors. In one of the circuits, all the capacitors are grounded. However, the circuit has the disadvantage of interdependent control of the frequency and the condition of oscillation. In two of the circuits, independent control of the frequency and the condition of oscillation can be achieved. However, the implementation of these two circuits requires floating capacitors and floating resistors. The fourth circuit 
requires floating capacitors and floating resistors and does not enjoy the independent control of the frequency and the condition of oscillation. Finally, in [12] a single-resistance-controlled sinusoidal oscillator is presented. The circuit exploits to advantage the internal poles of two CFOAs and uses three externally connected resistors; two of them are floating. While the frequency of oscillation can be controlled by adjusting a floating resistor without disturbing the condition of oscillation, the condition of oscillation can not be controlled without disturbing the frequency of oscillation.

It appears, therefore, that a sinusoidal oscillator circuit using the CFOA-pole and enjoying the attractive features of (i) independent control of the frequency of oscillation and the condition of oscillation by using grounded elements, (ii) use of externally connected grounded capacitors and resistors and (iii) low output impedance, is not available yet in the literature. It is the major intention of this paper is to present such an oscillator structure. The proposed structure exploits to advantage the internal poles of two CFOAs and uses grounded capacitors and resistors only.

\section{PROPOSED CIRCUIT}

The proposed oscillator circuit is shown in Figure 1. Figure 2 shows a simplified equivalent circuit for the CFOA. In this equivalent circuit the parallel combination of $C_{z}$ and $R_{z}$ represents the parasitic impedance at node $z$ of the CFOA, the parallel combination of $C_{y}$ and $R_{y}$ represents the parasitic impedance at node $y$ of the CFOA $\alpha=1-\varepsilon_{1},\left|\varepsilon_{1}\right| \ll 1$ represents the current-tracking error of the CFOA, $\beta=1-\varepsilon_{2},\left|\varepsilon_{2}\right| \ll 1$ represents the input voltage-tracking error, and $\gamma=1-\varepsilon_{3},\left|\varepsilon_{3}\right| \ll 1$ represents the output voltage-tracking error of the CFOA. Using the CFOA equivalent circuit of Figure 2, the equivalent circuit of the proposed oscillator structure of Figure 1, is shown in Figure 3. Routine analysis yields the characteristic equation of the circuit of Figure 1 which can be expressed as

$$
\left(G_{T_{1}}+s C_{T_{1}}\right)\left(G_{T_{2}}+s C_{T_{2}}\right)=\alpha_{1} \alpha_{2} \beta_{1} \beta_{2} \gamma_{1} \gamma_{2} Y_{1} Y_{2}
$$

where $G_{T_{1}}=G_{z_{1}}+G_{a}+G_{y_{2}}, G_{T_{2}}=G_{z_{2}}+G_{b}+G_{y_{1}}, G_{a}=\left(1 / R_{a}\right), G_{b}=$ $\left(1 / R_{b}\right), G_{z_{i}}=\left(1 / R_{z_{i}}\right), G_{y i}=\left(1 / R_{y_{i}}\right)$ are the internal conductances at 


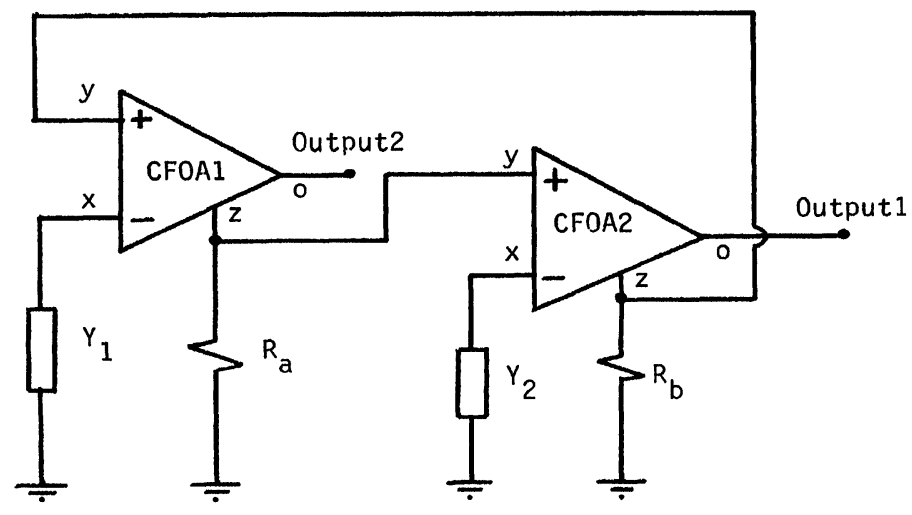

FIGURE 1 Proposed oscillator structure.
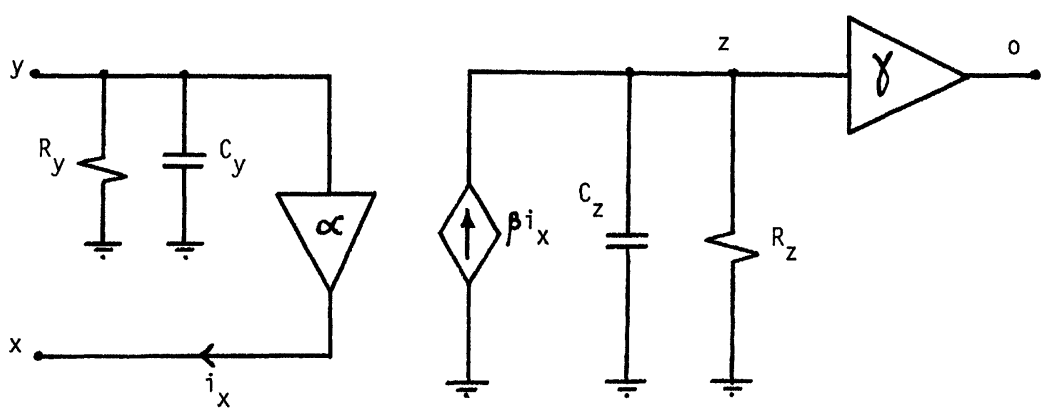

FIGURE 2 Equivalent circuit of the CFOA.

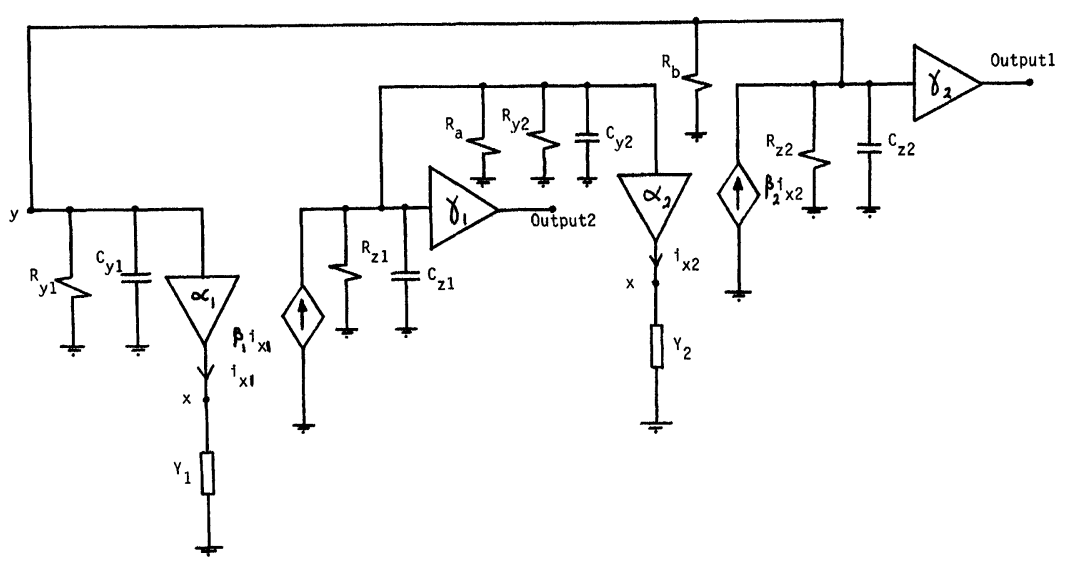

FIGURE 3 Equivalent circuit of the proposed oscillator structure. 
nodes $z$ and $y$ of the ith CFOA, and $\alpha_{i}, \beta_{i}$ and $\gamma_{i}$ are the current tracking-error, the input voltage tracking-error and the output voltage tracking-error of the ith CFOA. Using (1) several oscillator circuits can be derived from Figure 1. Table I summarizes the admittances, the frequency and the condition of oscillation of the resulting oscillator circuits using four or five passive elements only. However, for circuits 1-4, the resistors $R_{a}$ and $R_{b}$ can be removed and the design can account on the internal conductances of the CFOAs. Thus, circuits $1-$ 4 can be realised using three externally connected passive elements only.

From the Table it can be seen that for the circuits 1-4 the frequency of oscillation can be adjusted by tuning a single element (capacitor or resistor) without disturbing the condition of oscillation, while the condition of oscillation can be adjusted by tuning another element (resistor or capacitor) without disturbing the frequency of oscillation. Thus the circuits 1-4 enjoy independent oscillation and frequency control. For example, for circuit 4 , the frequency of oscillation can be adjusted by tuning $G_{1}$ without disturbing the condition of oscillation, while the condition of oscillation can be adjusted by tuning $C_{1}$ without disturbing the frequency of oscillation. However, because of the difference terms in the numerator (or denominator) of the oscillation frequency of the circuits $1-4$, it is essential to select components to satisfy the condition $C_{T_{1}} C_{T_{2}}>C_{1} C_{2}$ (or $G_{T_{1}} G_{T_{2}}>G_{1} G_{2}$ ).

TABLE I Frequency and Condition of Oscillation of the 6 Oscillators

\begin{tabular}{|c|c|c|c|c|}
\hline \multirow[t]{2}{*}{ Circuit } & \multicolumn{2}{|c|}{ Admittances } & \multirow{2}{*}{$\begin{array}{l}\text { Frequency of } \\
\text { Oscillation } W_{0}^{2}\end{array}$} & \multirow{2}{*}{$\begin{array}{c}\text { Condition of } \\
\text { Oscillation }\end{array}$} \\
\hline & $Y_{1}$ & $Y_{2}$ & & \\
\hline 1 & $s C_{1}$ & $G_{2}+S C_{2}$ & $\frac{G_{T_{1}} G_{T_{2}}}{C_{T_{1}} C_{T_{2}}-C_{1} C_{2}}$ & $\begin{array}{c}\alpha_{1} \alpha_{2} \beta_{1} \beta_{2} \gamma_{1} \gamma_{2} G_{2} C_{1}= \\
G_{T_{1}} C_{T_{2}}+G_{T_{2}} C_{T_{1}}\end{array}$ \\
\hline 2 & $G_{1+s} C_{1}$ & $s C_{2}$ & $\frac{G_{T_{1}} G_{T_{2}}}{C_{T_{1}} C_{T_{2}}-C_{1} C_{2}}$ & $\begin{array}{c}\alpha_{1} \alpha_{2} \beta_{1} \beta_{2} \gamma_{1} \gamma_{2} G_{1} C_{2}= \\
G_{T_{1}} C_{T_{2}}+G_{T_{2}} C_{T_{1}}\end{array}$ \\
\hline 3 & $G_{1}$ & $G_{2+} s C_{2}$ & $\frac{G_{T_{1}} G_{T_{2}}-\delta G_{1} G_{2}}{C_{T_{1}} C_{T_{2}}}$ & $\begin{array}{c}\alpha_{1} \alpha_{2} \beta_{1} \beta_{2} \gamma_{1} \gamma_{2} G_{1} C_{2}= \\
G_{T_{1}} C_{T_{2}}+G_{T_{2}} C_{T_{1}}\end{array}$ \\
\hline 4 & $G_{1+s} C_{1}$ & $G_{2}$ & $\frac{G_{T_{1}} G_{T_{2}}-\delta G_{1} G_{2}}{C_{T_{1}} C_{T_{2}}}$ & $\begin{array}{c}\alpha_{1} \alpha_{2} \beta_{1} \beta_{2} \gamma_{1} \gamma_{2} G_{2} C_{1}= \\
G_{T_{1}} C_{T_{2}}+G_{T_{2}} C_{T_{1}}\end{array}$ \\
\hline 5 & $s C_{1}$ & $G_{2}$ & $\frac{G_{T_{1}} G_{T_{2}}}{C_{T_{1}} C_{T_{2}}}$ & $\begin{array}{c}\alpha_{1} \alpha_{2} \beta_{1} \beta_{2} \gamma_{1} \gamma_{2} G_{2} C_{1}= \\
G_{T_{1}} C_{T_{2}}+G_{T_{2}} C_{T_{1}}\end{array}$ \\
\hline 6 & $G_{1}$ & $s C_{2}$ & $\frac{G_{T_{1}} G_{T_{2}}}{C_{T_{1}} C_{T_{2}}}$ & $\begin{array}{c}\alpha_{1} \alpha_{2} \beta_{1} \beta_{2} \gamma_{1} \gamma_{2} G_{1} C_{2}= \\
G_{T_{1}} C_{T_{2}}+G_{T_{2}} C_{T_{1}}\end{array}$ \\
\hline
\end{tabular}

$\delta=\alpha_{1} \alpha_{2} \beta_{1} \beta_{2} \gamma_{1} \gamma_{2}$ 
From the Table it can also be seen that circuits 5-6, although using fewer components, their frequency and condition of oscillation are interdependent. These circuits may be useful in applications where single-frequency oscillators are required. The frequency of oscillation of the circuits 5-6 can be adjusted by tuning the resistors $R_{a}$ and/or $R_{b}$.

\section{SENSETIVITY ANALYSIS}

By relating a sensetivity parameter $F$ to the element of variation $x_{i}$ by

$$
S_{x_{i}}^{F}=\frac{x_{i}}{F} \frac{d F}{d x_{i}}
$$

it is easy to show that the $\omega_{o}$-sensetivities of the circuits 5 and 6 can be expressed as

$$
\begin{aligned}
S_{G_{T_{1}}}^{\omega_{o}} & =S_{G_{T_{2}}}^{\omega_{o}}=-S_{C_{T_{1}}}^{\omega_{o}}=-S_{C_{T_{2}}}^{\omega_{o}} \\
S_{\alpha_{1}}^{\omega_{o}} & =S_{\alpha_{2}}^{\omega_{o}}=S_{\beta_{1}}^{\omega_{o}}=S_{\beta_{2}}^{\omega_{o}}=S_{\gamma_{1}}^{\omega_{o}}=S_{\gamma_{2}}^{\omega_{o}}=0
\end{aligned}
$$

Thus, the circuits 5 and 6 enjoy low active and passive $\omega_{o}$-sensetivities.

In the case of circuits $1-4$, the passive $\omega_{0}$-sensetivities may be appreciably higher due to the presence of the difference terms in the denominators. For example, for the circuits 1 and 2 , the $\omega_{o}$-sensetivities can be expressed as

$$
\begin{aligned}
S_{G_{T_{1}}}^{\omega_{o}} & =S_{G_{T_{2}}}^{\omega_{o}}=\frac{1}{2} \\
S_{C_{T_{1}}}^{\omega_{o}} & =S_{C_{T_{2}}}^{\omega_{o}}=\frac{-1}{1-\left(C_{1} C_{2} / C_{T_{1}} C_{T_{2}}\right)} \\
S_{C_{1}}^{\omega_{o}} & =S_{C_{2}}^{\omega_{o}}=\frac{-1}{\left(C_{1} C_{2} / C_{T_{1}} C_{T_{2}}\right)-1}
\end{aligned}
$$

and

$$
S_{\alpha_{1}}^{\omega_{o}}=S_{\alpha_{2}}^{\omega_{o}}=S_{\beta_{1}}^{\omega_{o}}=S_{\beta_{2}}^{\omega_{o}}=S_{\gamma_{1}}^{\omega_{o}}=S_{\gamma_{2}}^{\omega_{o}}=0
$$

Thus, while the active $\omega_{o}$-sensetivities of the circuits 1 and 2 are zeros, the passive sensitivities may be appreciably high. However, careful 
selection of the passive components can yield passive $\omega_{o}$-sensetivities of the same order as those of circuits 5 and 6.

Finally, the $\omega_{o}$-sensetivities of the circuits $3-4$ can be expressed as

$$
\begin{aligned}
S_{C_{T_{1}}}^{\omega_{o}} & =S_{C_{T_{2}}}^{\omega_{o}}=-\frac{1}{2} \\
S_{G_{T_{1}}}^{\omega_{o}} & =S_{G_{T_{2}}}^{\omega_{o}}=\frac{1}{1-\left(\delta G_{1} G_{2} / G_{T_{1}} G_{T_{2}}\right)} \\
S_{G_{1}}^{\omega_{o}} & =S_{G_{2}}^{\omega_{o}}=S_{\delta}^{\omega_{o}}=\frac{1}{1-\left(G_{T_{1}} G_{T_{2}} / \delta G_{1} G_{2}\right)}
\end{aligned}
$$

Thus, circuits 3 and 4 has non-zero active $\omega_{o}$-sensetivities and its passive $\omega_{o}$-sensetivities may be appreciably high. However, careful design may result in active and passive $\omega_{o}$-sensetivities of the same order as those of circuits 5 and 6 .

\section{EXPERIMENTAL RESULTS}

The proposed sinusoidal oscillator circuits were experimentally tested using the AD844 CFOA. The results obtained from circuits 3-6 are shown in Figure 4. Because of the large values of stray capacitances encountered in a breadboard implementation, it is difficult to successfully obtain oscillations from circuits 1 and 2 . Extra care must be taken to minimize the effect of the stray capacitances. Thus, circuits 1 and 2 are more suitable for integrated circuit implementation.

For the $\mathrm{AD} 844$ the capacitances $C_{z} \cong 5 \mathrm{pF}, C_{y} \cong 3 \mathrm{pF}$ and the resistances $R_{z}=3 \mathrm{M} \Omega, R_{y}=10 \mathrm{M} \Omega$. However, the total parasitic capacitance between terminals $Z$ of the AD844 and the breadboard was measured to be about $25 \mathrm{pF}$. Thus $C_{T_{1}}=C_{T_{2}}$ was taken as $33 \mathrm{pF}$. The experimental results confirm the validity of the proposed oscillator structure for generating high frequencies.

It is worth mentioning, however, that the experimental results obtained from circuits 5 and 6 are different even for same values of externally connected resistances. This is attributed to the influence of the resistance $R_{x}$ at terminal X of the $\mathrm{AD} 844$. This resistance is about $50 \Omega$ and when a capacitance is connected to terminal $\mathrm{X}$ this resistance 


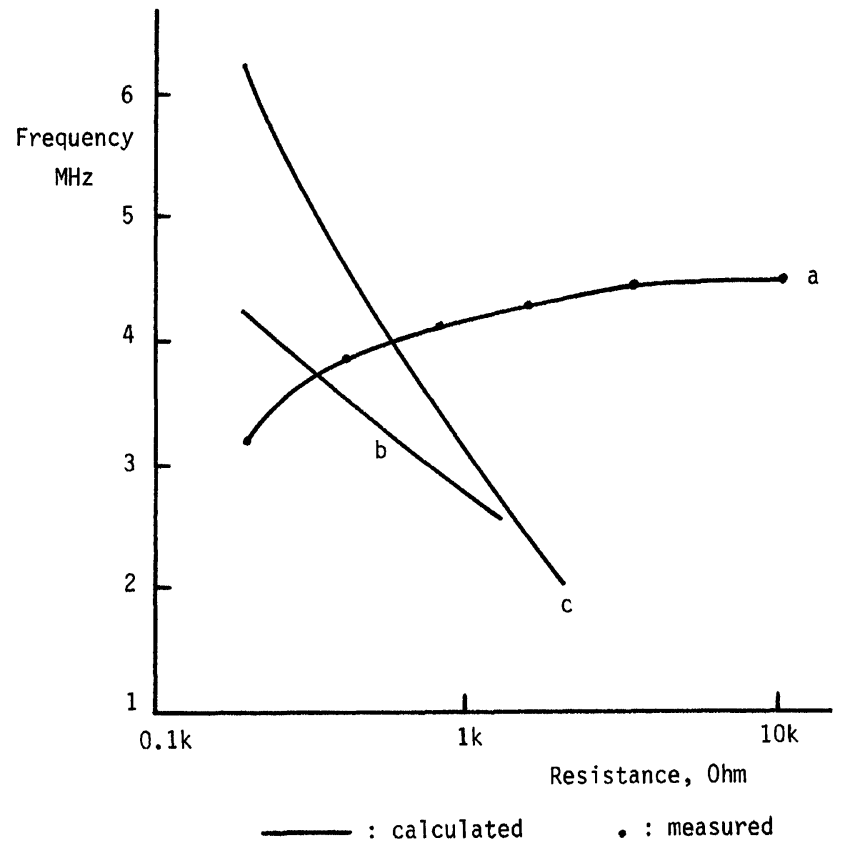

FIGURE 4 Measured and calculated results obtained from. (a) Circuits 3, 4 with: $R_{a}=R_{b}=1 \mathrm{~K} \Omega, R_{2}=10 \mathrm{~K} \Omega, C_{1}=C_{2}=100 \mathrm{pF}, R_{1}=200 \Omega-10 \mathrm{~K} \Omega$; (b) Circuit 5 with: $R_{2}=10 \mathrm{~K} \Omega . R_{b}=1 \mathrm{~K} \Omega, C_{1}=470 \mathrm{pF}, R_{a}=200 \Omega-800 \Omega$; (c) Circuit $b$ with: $R_{b}=1 \mathrm{~K} \Omega$, $R_{1}=10 \mathrm{~K} \Omega, C_{2}=300 \mathrm{pF}, R_{a}=200 \Omega-2 \mathrm{~K} \Omega$.

will affect the transfer function especially at high frequencies [13]. Thus, with $C_{1}=470 \mathrm{pF}$ for circuit 5 and $C_{2}=300 \mathrm{pF}$ for circuit 6 , the experimental results are different at high frequencies. As the frequency decreases the results obtained from the two circuits becomes closer to each other. This effect is not observed in circuits 3 and 4. This is attributed to the symmetry between the two circuits regarding the externally connected elements to terminals X. Moreover, circuits 3 and 4 use relatively lower value for capacitances, $C_{1}=C_{2}=100 \mathrm{pF}$. Thus, the effect of $R_{x}$ would manifest itself at relatively higher frequencies.

In order to minimize the effect of the parasitic resistance $R_{x}$ on the circuit performance, it is recommended here to use CFOAs designed using the power-supply current-sensing technique, rather than the AD844 which is built around the translinear principle [13] and suffers from relatively large value of the parasitic resistance $R_{x}$. 


\section{CONCLUSION}

New grounded-capacitor grounded-resistor CFOA-pole-based sinusoidal oscillator circuits have been presented. Each circuit uses two CFOAs, at most five passive elements and exploits, to advantage, the internal poles of the CFOAs. Some of the circuits enjoy independent control of the frequency and the condition of oscillation. The use of grounded capacitors and resistors is an attractive feature for integration. Moreover, each circuit has two low impedance outputs. Thus, in comparison to similar ideal current-conveyor-based topologies [14], the proposed circuits enjoy the attractive features of using less number of passive components while providing two lowimpedance outputs and exploiting, to advantage, the nonidealities of the active devices.

\section{References}

[1] Abuelma'atti, M. T. and Almansoury, W. A., Identification of two-amplifier activeR sinusoidal oscillators, IEEE Proceedings, 134, Part G, 137-140.

[2] Battacharyya, B. B. and Natarajan, S. (1977). A new continuously tuneable sinusoidal oscillator without external capacitors, IEEE Proceedings, 65, 17261727.

[3] Stiurca, D. (1994). On the multiphase symmetrical active-R oscillators, IEEE Transactions on Circuits and Systems-II: Analog and Digital Signal Processing, 41, $156-158$.

[4] Liu, S.-I., Chang, C.-C. and Wu, D.-S. (1994). Active-R sinusoidal oscillators using the CFA pole, International Journal of Electronics, 77, 1035-1042.

[5] Wu, D.-S., Liu, S.-I., Hwang, Y.-S. and Wu, Y.-P. (1995). Multiphase sinusoidal oscillator using the CFOA pole, IEEE proceedings-Circuits Devices Systems, 142, $37-40$

[6] Senani, R. and Singh, V. K. (1996). Synthesis of canonic single-resistancecontrolled-oscillators using a single current-feedback-amplifier, IEEE Proceedings-Circuits, Devices and Systems, 143, 71-72.

[7] Toumazou, C., Payne, A. and Pookaiyaudom, S. (1995). The active-R filter technique applied to current-feedback op-Amps, Proceedings of the International Symposium on Circuits and Systems, pp. 1203-1206.

[8] Payne, A. and Toumazou, C. (1995). Analogue circuit design using currentfeedback active-R op-amps, Electronic Engineering, 67, 77-78, 80.

[9] Abuelma'atti, M. T. and Al-Shahrani, S. M. (1996). A novel low-component-count single-element-controlled sinusoidal oscillator using the CFOA pole, International Journal of Electronics, 80, 747-752.

[10] Martinez, P. A., Celma, S. and Sabadell, J. (1996). Designing sinusoidal oscillators with current-feedback amplifiers, International Journal of Electronics, 80, 637-646.

[11] Abuelma'atti, M. T., Farooqi, A. A. and Al-Shahrani, S. M. (1996). Novel RC oscillators using the current-feedback operational amplifier, IEEE Transactions on Circuits and Systems-I: Fundamental Theory and Applications, 43, 155-157. 
[12] Senani, R. and Singh, V. K. (1996). Single-resistance-controlled-oscillator configuration using current feedback amplifiers, IEEE Transactions on Circuits and Systems-I: Fundamental Theory and Applications, 43, 698-700.

[13] Fabre, A., Saaid, O., Wiest, F. and Boucheron, C. (1996). High frequency applications based on a new current controlled conveyor, IEEE Transactions on Circuits and Systems-I: Fundamental Theory and Applications, 43, 82-91.

[14] Abuelma'atti, M. T., Al-Ghumaiz, A. A. and Khan, M. H. (1995). Novel CCIIbased single-element controlled oscillators employing grounded resistors and capacitors, International Journal of Electronics, 78, 1107-1112. 

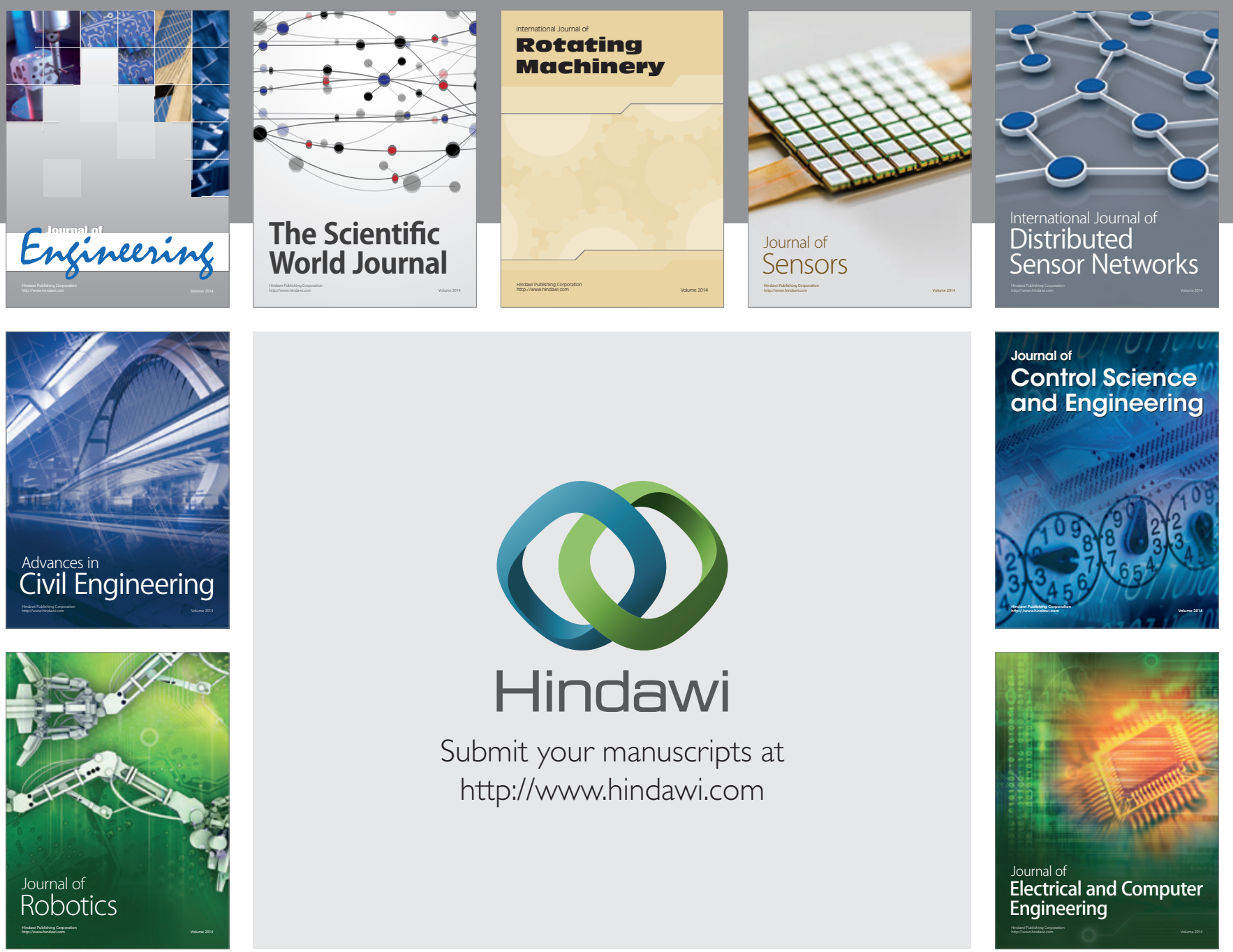

Submit your manuscripts at

http://www.hindawi.com
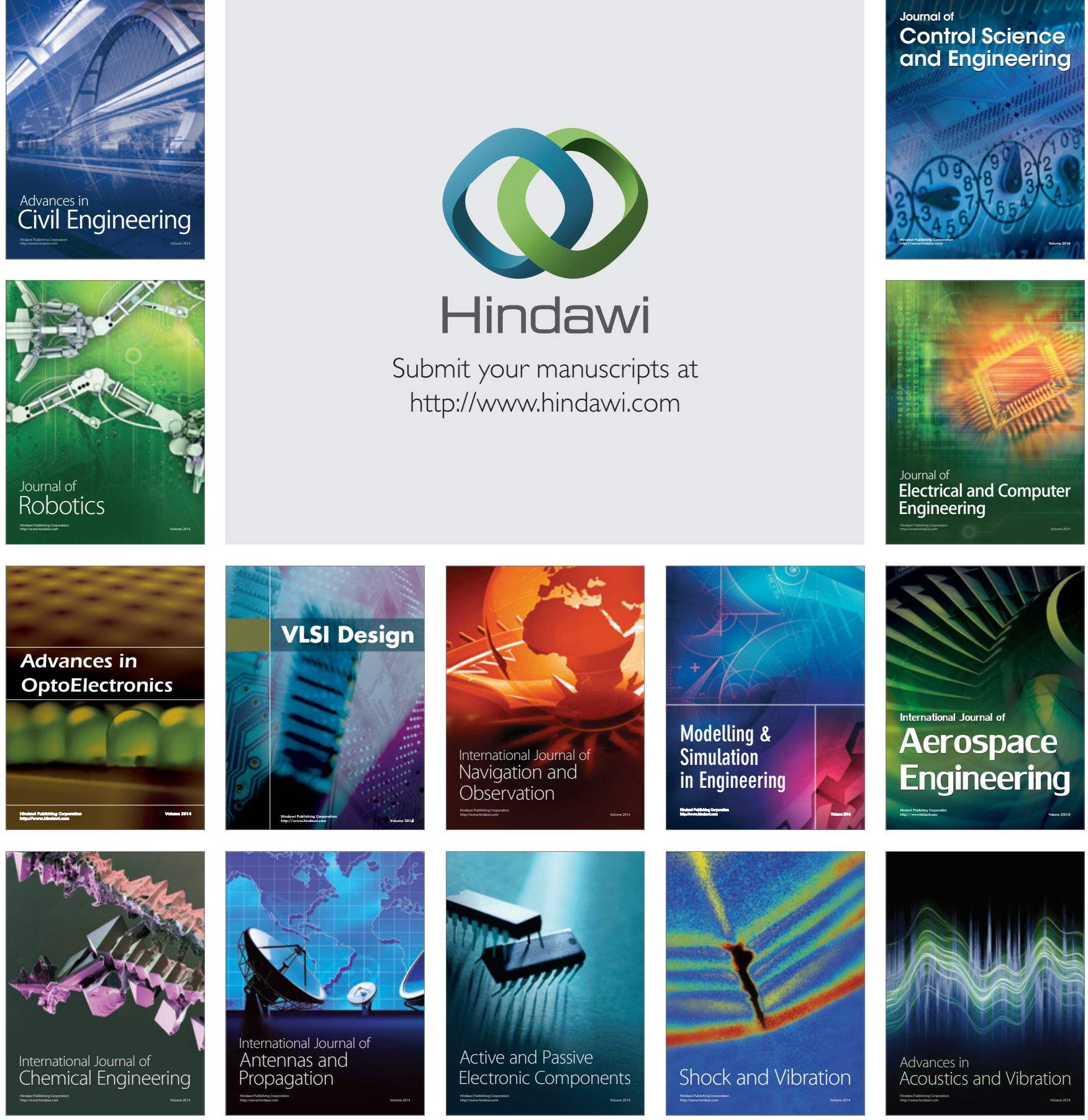